\title{
Biden y América Latina. Desafíos regionales para la política exterior estadounidense
}

\author{
Javier Luciano Quispe Robles*
}

\begin{abstract}
RESUMEN
¿Cuáles son los desafíos que enfrenta la política exterior de Joe Biden en América Latina? El presidente nortemericano ha prometido abrir una nueva etapa en las relaciones con los países latinoamericanos luego de cuatro ańos de una política exterior unilateral e impredecible de Donald Trump. Sin embargo, existen procesos en marcha en América Latina que imponen condiciones importantes para la política exterior estadounidense: procesos de autocratización en varios países de la región, una política exterior autónoma y competitiva de parte de las potencias regionales latinoamericanas y la creciente presencia de China en la región. El artículo discute cómo estos procesos configuran la política regional latinoamericana y qué desafíos representan para la amplia agenda que tiene Biden para la región, que va desde asuntos ambientales, migración, corrupción, derechos humanos, entre otros. Para ilustrar el argumento, se analiza la relación de Estados Unidos con países particulares a la luz de acontecimientos recientes. Así, el artículo busca contribuir con la discusión sobre factores que inciden en la política exterior y en específico en la relación de Estados Unidos con América Latina.
\end{abstract}

Palabras clave: América Latina, Estados Unidos, política exterior, autocratización, potencias regionales.

\footnotetext{
Estudiante del Doctorado en Ciencia Política de la Pontificia Universidad Católica de Chile. Licenciado en Ciencia Política y Gobierno por la Pontificia Universidad Católica del Perú y magíster en Ciencia Política por el Centro de Investigación y Docencia Económicas (CIDE) de México. Posee un diplomado en Relaciones Internacionales por la Academia Diplomática del Perú. Correo electrónico: lquispe1@uc.cl
} 
Biden and Latin America. Regional Challenges for US Foreign Policy

\section{Abstract}

What are the challenges Joe Biden's foreign policy faces in Latin America? The American president has promised to open a new era in foreign relations with Latin American countries after four years of Trump's unilateral and unpredictable foreign policy. However, there are processes underway in Latin America that impose significant constraints for US foreign policy: processes of democratic erosion in several countries of the region, a competitive regional environment led by regional Latin American powers, and a growing presence of China in the region. This article discusses how these processes shape Latin American regional politics and how they challenge the Biden's broad agenda, ranging from environmental issues, migration, corruption, and human rights. Finally, the article analyzes the engagement of the United States with Latin American countries in light of recent events. Thus, the paper seeks to contribute to the discussion on factors that affect foreign policy and the relationship of the United States with Latin America.

Keywords: Latin America, United States, foreign policy, autocratization, regional power

La victoria del candidato demócrata Joe Biden en noviembre de 2020 generó mucho optimismo a nivel internacional luego de cuatro años de una política exterior errática e incoherente en términos estratégicos de parte del gobierno de Donald Trump. América Latina, en particular, fue uno de los tantos escenarios internacionales en donde la administración republicana generó tensiones, provocó conflictos diplomáticos, estropeó alianzas bien establecidas y generó incertidumbre con decisiones unilaterales. Durante la campaña presidencial, y ya como presidente, Joe Biden ha manifestado públicamente su intención de renovar la política exterior estadounidense hacia América Latina, en vista de los problemas urgentes que enfrenta la región y que afectan directamente al país norteamericano. Sin embargo, ¿qué tan factible es un viraje completo de la política exterior? ¿Cuáles son las posibilidades y límites que la región le plantea a los Estados Unidos?

Este artículo sugiere que la política exterior de Biden hacia la región se encuentra condicionada por un conjunto de procesos políticos regionales que definirían su alcance en los próximos años: en primer lugar, los procesos de autocratización en la región, un fenómeno que limita la efectividad de la política exterior para lidiar con los problemas de la región como la violencia o la migración forzada; en segundo lugar, el comportamiento competitivo de las potencias regionales latinoamericanas, en específico países como Brasil, México y Argentina, líderes regionales con agenda propia y con capacidad para ejercer un balance efectivo a potencias extra regionales; finalmente, la presencia de China, un actor global que ha establecido rela- 
ciones estratégicas a nivel económico y de seguridad con distintos países de la región. $\mathrm{Si}$ bien estos procesos no se inauguran con Biden (las administraciones Obama y Trump lidiaron con ellos, con resultados variados), se han ido profundizando en los últimos ańos, configurando el escenario regional latinoamericano. Por ende, la Administración Biden debe tener en consideración este contexto a la hora formular y ejecutar la política exterior de su país.

Asimismo, es importante resaltar que América Latina experimenta estas transformaciones en consonancia con otros fenómenos de corte global, como la crisis del orden liberal internacional y la emergencia sanitaria a raíz del Covid-19. La política exterior estadounidense se enfrenta así a una región con una situación política muy compleja, con problemas crecientes cada vez más difíciles de solucionar. El artículo sugiere prestar atención a factores políticos en América Latina sin desmerecer la importancia de otros factores igual de relevantes como la economía internacional o procesos políticos al interior de Estados Unidos. En ese sentido, más que proveer una descripción comprehensiva de la política exterior estadounidense, el artículo busca contribuir con la discusión sobre qué factores podrían marcar la pauta de las relaciones entre Estados Unidos y la región en los próximos cuatro años.

El artículo presenta, en primer lugar, el interés y los objetivos de la política exterior estadounidense y en concreto Joe Biden en América Latina, así como se describen los procesos que configuran el panorama político en la región. Estos tres procesos son posteriormente desarrollados y ejemplificados con casos particulares, a la luz de los eventos ocurridos en los primeros meses del gobierno de Biden. Finalmente, se presentan conclusiones al respecto.

\section{La política exterior de Biden hacia Latinoamérica}

¿Qué busca lograr la política exterior de Joe Biden hacia América Latina? ¿Realmente propone un cambio significativo o apuesta por la continuidad? La política exterior de Biden hacia la región en sus primeros cien días dieron muestra de cuáles serían sus prioridades: se han observado iniciativas importantes en el manejo de la crisis migratoria centroamericana y la lucha contra el cambio climático. Sin embargo, en un contexto marcado por la emergencia sanitaria global a raíz del Covid-19, la región, no ha recibido suficiente atención en vista de las urgencias domésticas. Por supuesto, esto no significa que Biden no tenga objetivos con respecto a América Latina para los próximos años, sobre todo considerando el legado, en términos de Biden, desastroso para el liderazgo norteamericano, que hereda de su antecesor, el presidente Donald Trump (Biden, 2019). 
Es importante observar que las administraciones anteriores se caracterizaron por tener como prioridad de su política exterior otras regiones como Medio Oriente o el Asia-Pacífico, por los desafíos que el terrorismo internacional, Irán y China representan para el liderazgo estadounidense. Durante las dos últimas administraciones hubo pocas visitas de estado a la región latinoamericana y pocas iniciativas a escala regional. Proyectos como el Área de Libre Comercio para las Américas impulsado por el gobierno de George Bush fracasaron, en parte por el surgimiento de gobiernos de izquierda con propuestas divergentes en el plano político y económico, que evidenciaron el nivel de desconexión creciente entre Estados Unidos y la región (Williams, 2014). En el transcurso de los años, Estados Unidos lograría estrechar la relación con aliados tradicionales para la lucha contra el narcotráfico y el crimen organizado, contener el avance del denominado Socialismo del Siglo XXI e inaugurar nuevos episodios en la región como la reapertura de las relaciones con Cuba. Joe Biden como vicepresidente de Barack Obama a lo largo de ocho años participó en muchas de estas iniciativas. Sin embargo, los años de Trump complicaron significativamente las relaciones al carecer de coherencia estratégica, estropeando alianzas bien establecidas, generando incertidumbre con decisiones unilaterales, agudizando innecesariamente viejas rivalidades y debilitando la legitimidad del país, esencial para ejercer el liderazgo regional (Long, 2018; Romano, 2018).

En ese sentido, Biden ha señalado en numerosas ocasiones que busca iniciar una nueva era en las relaciones con América Latina. En un artículo titulado «Why America Must Lead Again», Biden planteó su visión del mundo de cara a los próximos años; sin embargo, las referencias a América Latina fueron escasas, evidenciando la poca importancia de la región frente a asunto más trascendentales como la contención a China, Rusia, el terrorismo internacional, la OTAN, entre otros (Biden, 2019). La referencia más importante con respecto a América Latina se encuentra relacionada con la seguridad interna y fronteriza del país: el control del flujo migratorio proveniente de Centroamérica, la migración no regular y los desafíos que representan para la seguridad del país. Para ello, Biden proponía repotenciar con un fondo histórico de 4 mil millones dólares la política de asistencia económica, financiera y técnica para luchar contra el flagelo de la corrupción, la violencia y la pobreza, en coordinación con los gobiernos de dicha región, al mismo tiempo que planteaba retraer todas las políticas de control migratorio que el presidente Trump había puesto en marcha y que habían incurrido en flagrantes violaciones de derechos humanos (Biden, 2019).

Las referencias de Biden a América Latina, aparte de las concernientes al asunto migratorio, han sido poco articuladas, generando en algunos casos reacciones airadas de parte de líderes como Jair Bolsonaro, aliado de su antecesor Donald Trump, que lo criticó duramente por sugerir una intervención en Brasil a raíz de la deforestación 
en la Amazonía, o de Nicolás Maduro luego de que Biden lo llamara «dictador» y asegurara el reconocimiento de Juan Guaidó como presidente. Por otro lado, en lo que respecta a asuntos económicos, Biden también ha prometido continuar la profundización de las relaciones comerciales con México en el marco del T-MEC y aun queda pendiente saber si promoverá el retorno de Estados Unidos al Acuerdo Transpacífico de Cooperación Económica, de mucha importancia para algunas economías latinoamericanas como México, Perú y Chile. Algunas de estas propuestas han sido ampliamente respaldadas por la vicepresidenta Kamala Harris, sobre todo la concerniente a la desmilitarización de la política migratoria, la seguridad fronteriza y la protección medioambiental (Nagovitch 2020). Por su parte, el secretario de Estado, Anthony Blinken, ha propuesto trabajar directamente con los gobiernos centroamericanos para combatir los problemas de crimen organizado, desempleo y corrupción, así como crear un mecanismo de distribución de vacunas para la región. Además, Biden ha nombrado además al ex embajador de Estados Unidos en Perú, Brian Nichols, como subsecretario de Estado para Asuntos del hemisferio occidental. La amplia experiencia de Nichols en la región buscaría ser aprovechada por Biden para lidiar con asuntos complejos como el de Venezuela y Cuba, en los que Trump ha tenido poco éxito (Gramer, 2021).

\section{Procesos políticos y los desafíos para Biden en la región}

La agenda exterior de Biden en Latinoamérica es bastante amplia, y desarrollarla temáticamente excede el alcance de este artículo; aun así, resulta bastante evidente que busca inaugurar una nueva relación con la relación para «enmendar» el legado que deja su antecesor. Para la realización de dicha agenda, sin embargo, es crucial que el gobierno de Biden considere los procesos de carácter político que viene atravesando la región y que vienen configurando un nuevo escenario. Estos procesos son entendidos como transformaciones políticas en marcha a nivel regional, que configuran el escenario sobre el que la política exterior se desenvolverá y afectan directamente las posibilidades de cooperación y presión que Biden busca lograr en la región. En concreto se consideran tres: los procesos de autocratización en la región, principalmente en Centroamérica; la agenda y estrategias de balance de las potencias latinoamericanas; y la creciente presencia de China en América Latina. Estos procesos no han iniciado recientemente, puesto que los gobiernos de Bush, Obama y Trump han tenido que lidiar con versiones anteriores de ellos; sin embargo, Biden los enfrenta en un nuevo escenario el que el prestigio estadounidense está profundamente dañado en la región. A continuación, se desarrollan estos tres procesos, evaluando el desafío que representan para la administración Biden en los próximos cuatro ańos y a la luz de hechos recientes. 


\section{Autocratización en Centroamérica}

En primer lugar, la región experimenta lo que algunos autores han identificado como recesión democrática o procesos de autocratización en ciernes, es decir la pérdida progresiva de elementos constitutivos de todo régimen democrático como la participación política, la competencia por el poder y la realización de elecciones libres y justas (Levitsky y Way, 2015; Lürhmann y Lindberg, 2018). Los índices de democracia según el reporte de Freedom House para el año 2021 muestran un retroceso importante en América Latina, encabezado por el propio Estados Unidos (Freedom House, 2021). Gran parte de la agenda que Biden busca desarrollar en América Latina depende del funcionamiento de las instituciones democráticas y el estado de derecho en la región, como la lucha contra la corrupción y la violencia. Sin embargo, los últimos gobiernos estadounidenses no han sabido lidiar con el surgimiento de autoritarismos y autoritarismos competitivos en la región, sino que en algunos casos los han promovido y reforzado con apoyo material y político (Romano, 2018). Este proceso de autocratización se ha manifestado con fuerza en los últimos años en Centroamérica, y es importante observar las implicancias de este fenómeno en esta región pues se encuentra estrechamente ligada con quizás el desafío más importante y próximo para Biden en la región: la crisis migratoria.

Para Estados Unidos, el punto más crítico de su agenda latinoamericana en vista de sus efectos en el orden doméstico es el de la migración proveniente desde el Triángulo Norte de Centroamérica (Guatemala, El Salvador y Honduras). En los primeros 100 días de su gobierno, Biden ha debido lidiar directamente con esta situación, reforzando el control en la frontera con México e imponiendo más restricciones en el acceso, a pesar de que había prometido lo contrario en campaña. Asimismo, ha continuado con la política de sus antecesores de brindar asistencia económica a los países centroamericanos con el fin de combatir las causas que motivan la movilidad forzada (Solís, 2021). Finalmente, Biden ha establecido una política de cooperación con México para reducir los flujos de migrantes a través de proyectos de inversión y desarrollo. Sin embargo, hay un trasfondo político que Washington debe considerar para el éxito o no de estas políticas: los procesos de autocratización en marcha en la región centroamericana.

Entre las razones que explican la migración forzada centroamericana se encuentran la pobreza y la violencia que experimentan estos países (Solís, 2021; Chappell, 26 de marzo 2021). Estos factores se han visto profundamente agudizados por la ineficiencia de sus gobiernos para hacerles frente pero también por las dinámicas autoritarias en los países de la región en los últimos años, que han debilitado a las instituciones encargadas de mantener el orden social, así como agudizado el problema 
de la corrupción. Este es un desafío directo para la administración estadounidense pues el apoyo financiero no llega a ser efectivo por estas razones. De acuerdo con Solís (2021), entre 2016 y 2021 el Congreso de Estados Unidos otorgó más de 3,6 mil millones de dólares a los países centroamericanos como parte de su Strategy for Engagement in Central America, para fortalecer programas sociales, fortalecer la lucha contra la corrupción y combatir el crimen organizado. Cinco años después, los niveles de pobreza, desempleo y violencia solo se han incrementado, evidenciando que la asistencia económica no es suficiente, lo cual pone en duda la efectividad de los 4 mil millones de dólares prometidos por la Administración Biden. Este hecho se encuentra vinculado al deterioro institucional de la región a raíz de la regresión democrática que experimenta la región.

La institucionalidad democrática en Centroamérica ha sido por lo general muy débil, pero hechos recientes como elecciones fraudulentas, persecución política, impunidad y casos de corrupción a gran escala muestran un reverso significativo del nivel de democracia en la región. De acuerdo al último reporte de Libertad en el Mundo de Freedom House (2021), Honduras, Guatemala y El Salvador muestran un retroceso significativo en sus indicadores sobre libertad y democracia y son considerados regímenes híbridos. Nicaragua es el único país en la región considerado como plena autocracia mientras que Costa Rica y Panamá son democracias plenas. Así, el debilitamiento de las instituciones democráticas ha conllevado a la concentración de poder en manos de líderes con poca voluntad de cambio y que se han mostrado profundamente ineficientes para atender los problemas de violencia, desempleo y corrupción en sus países. En marzo de 2021, Juan Gonzales, asesor de Biden para asuntos migratorios señaló que los países centroamericanos se encuentran cooptados por una élite depredadora que busca mantener el status quo en sus países, lo cual evidencia que la administración Biden es consciente de las raíces del problema a enfrentar (Chappell, 26 de marzo 2021).

El caso de Honduras es uno de los más dramáticos, pero además ilustra el desempeño ineficiente de la política exterior estadounidense en la región. El presidente Juan Orlando Hernández obtuvo la reelección en 2017 en un proceso fraudulento que contó con el respaldo del gobierno de Donald Trump, quien consideró a Hernández un aliado político importante para manejar el problema migratorio y establecer, junto con Guatemala y El Salvador los acuerdos de Tercer País Seguro, que permitiría deportar inmigrantes no regulares a dichos países. La política de militarización de Hernández para la lucha contra el crimen organizado y el manejo de los flujos migrantes ha contado con el apoyo político y económico de los Estados Unidos, con resultados poco auspiciosos y múltiples denuncias de violaciones a los derechos humanos y asesinatos de líderes sociales (Romano, 2018). 
Un problema que Honduras y Guatemala comparten es el de la corrupción y el involucramiento directo de sus presidentes y altos funcionarios. Además de Hernández, los presidentes guatemaltecos Otto Pérez Molina y Jimmy Morales han sido vinculados a casos de corrupción y narcotráfico. A raíz de los pocos esfuerzos de parte de las administraciones nacionales para hacer frente a este problema, se instalaron en ambos países misiones internacionales como la Comisión Internacional contra la Impunidad en Guatemala (CICIG) en diciembre de 2006 y la Misión de Apoyo contra la Corrupción y la Impunidad en Honduras (MACCIH) en enero de 2016, coordinadas con las Naciones Unidas y la OEA respectivamente. Ambas instituciones han logrado avances importantes para la investigación, denuncia y procesamiento de altos funcionarios, aunque han sido ampliamente resistidas y cuestionadas por sectores poderosos de cada país. En 2018 la CICIG sería expulsada del país por el presidente Morales, a quien la comisión acusaba de prácticas ilícitas y para quien solicitaba el desafuero; mientras que en 2019 Hernández decidiría repentinamente no renovar el convenio para renovar la misión.

Por otro lado, El Salvador de Nayib Bukele presenta otro desafío político para Biden debido al débil respeto que el presidente salvadoreño ha mostrado frente a las instituciones democráticas del país como el parlamento y las instituciones judiciales. Asimismo, el partido Nuevas Ideas de Bukele obtuvo una mayoría absoluta en las elecciones parlamentarias de 2021 y ha mostrado, hasta el momento, un débil compromiso con la independencia de poder al promover activamente la destitución de los jueces de la Sala de lo Constitucional de la Corte Suprema de Justicia en mayo del mismo ańo. De acuerdo al reporte de Freedom House de 2021, la pandemia ha reafirmado esas tendencias autoritarias, que además se han expresado en un incremento de violaciones de derechos humanos y los abusos de la policía y militares. Bukele había mantenido además un diálogo constante con la administración Trump, por lo que el perfil político de su gobierno y la inmensa concentración de poder a nivel doméstico del que goza representa un desafío para Washington.

Por otro lado, la Nicaragua de Ortega, en el poder desde 2007, es el caso de regresión democrática más flagrante en el istmo centroamericano. Si bien la contribución de Nicaragua a la migración forzada hacia el norte es menor, la crisis económica que experimenta el país en conjunto con la represión estatal, la persecución política y el uso de la violencia indiscriminada contra la población pueden conllevar a una explosión social con consecuencias humanitarias desastrosas. La presión internacional ante esta situación se ha visto estropeada por la inoperatividad política de organismos regionales, por la protección de regímenes autoritarios como Venezuela y China han brindado a Ortega, y por la falta de una estrategia coherente de parte de Estados Unidos para lidiar con este asunto. El gobierno de Biden tendrá como prueba de 
fuego las elecciones generales de noviembre de 2021 en las que Ortega postulará nuevamente.

Además de Centroamérica, existen otros casos de autoritarismo en la región que plantean desafíos a la política exterior de Biden, aunque no al mismo nivel de urgencia que Centroamérica. Venezuela y Cuba son los casos más significativos de autoritarismo con los Estados Unidos ha lidiado y de forma poco exitosa en los últimos años. Las sanciones impuestas a ambos países se han incrementado durante el gobierno de Trump, han estropeado los avances hechos por administraciones anteriores y no existen mayores indicios de que Biden vaya a adoptar una estrategia diferente. Un punto señalado por Long (2021) ayudaría a explicar el cálculo de Biden: adoptar una línea más pragmática resulta electoralmente costoso en Florida, donde la oposición a los regímenes cubanos y venezolanos es activa. Dado que el Partido Demócrata perdió Florida en las elecciones de 2020, buscaría adoptar una postura dura con ambos países, con el objetivo de recuperar el voto latino, conformado por las diásporas provenientes de ambos países.

Finalmente, otros dos casos merecen mención: México y Brasil, bajo los gobiernos de López Obrador y Bolsonaro han experimentado también un debilitamiento de sus instituciones democráticas, principalmente las entidades electorales, las instituciones fiscalizadoras y los jueces. Este es un riesgo potencial que la Administración Biden debería considerar teniendo en cuenta el peso relativo de ambos países en la región. Sobre este punto trata el siguiente apartado.

\section{La agenda y estrategias de las potencias latinoamericanas}

Las potencias regionales latinoamericanas son actores fundamentales para llevar a cabo los objetivos que se plantea Estados Unidos para la región; sin embargo, estas han mostrado un nivel importante de autonomía en los últimos años frente a la voluntad de Estados Unidos y otras potencias en la región (Malamud, 2011; Flemes y Wehner, 2012; Gardini, 2016). La literatura ha identificado que los países más débiles recurren a distintas herramientas y estrategias de política exterior para ejercer poder frente a adversarios más poderosos, limitando así la capacidad de estos para ejercer presión e influencia sobre ellos (Long, 2015). Asimismo, las potencias regionales compiten intensamente entre sí usando estrategias de poder duro y blando para contener a sus potenciales rivales y ganar preeminencia en la región. América Latina no es una región ajena a estas dinámicas, que pueden observarse en el comportamiento de Brasil, México, Argentina, Venezuela y Colombia en las últimas décadas (Schenoni, 2017). La distribución de poder regional y las capacidades de estas potencias son factores que imponen restricciones a la política exterior estadounidense, que 
en muchas ocasiones se ha visto limitada para condicionar el comportamiento de estados militar y económicamente más débiles.

En un análisis muy sugerente, Long (2021) plantea que la ausencia de un claro liderazgo en Latinoamérica dificulta la política exterior estadounidense hacia la región, dado que conlleva a la fragmentación política de la región, expresada por ejemplo en el colapso de instituciones regionales como UNASUR o el debilitamiento de CELAC, esenciales para el diálogo y la cooperación; así como en la ausencia de una estrategia regional coordinada para lidiar con desafíos regionales como el manejo de la pandemia. ¿Cuáles son las implicancias políticas de la ausencia de una potencia regional? Cuando se discute de liderazgo regional latinoamericano, el referente más cercano es Brasil, país que durante los gobiernos de Lula buscó claramente ejercer un liderazgo político en la región, apoyado en su ascenso económico y su posicionamiento como la séptima economía global (Malamud, 2011; Gardini, 2016). Dicho ímpetu se estancaría debido al estancamiento de la economía, a los casos de corrupción y a la crisis política, deviniendo finalmente en un viraje de política exterior.

Lo anterior plantea importantes desafíos para la política exterior estadounidense. Durante los años de Obama, Brasil y Estados Unidos mantuvieron una relación bastante positiva que luego se vería reforzada con la llegada al poder de Donald Trump y Jair Bolsonaro, en vista de las coincidencias ideológicas y el abandono de Brasil de una política exterior enfocada en el liderazgo regional. Al mismo tiempo, el peso relativo de Brasil es importante en términos económicos y militares, lo cual determina los cálculos de Estados Unidos a la hora de vincularse con un país que puede resultar o un contrincante importante en la propia región o un aliado muy importante (Weyland, 2016).

Un desafío que ilustra lo anterior son los incendios en la Amazonía. Joe Biden expuso desde la campaña presidencial la necesidad de ejercer una mayor presión internacional sobre Brasil para lidiar con los incendios forestales y la deforestación amazónica, lo cual motivó una airada reacción diplomática brasileña. En efecto, como señala Walt (2019), la deforestación amazónica representa un riesgo no regional sino global que ameritan una respuesta de la comunidad internacional y sobre todo los Estados Unidos. Sin embargo, una vez en el poder, Biden ha buscado establecer una relación más pragmática con Brasil para evitar una confrontación con el país latinoamericano más poderoso y al mismo tiempo impulsar su agenda ambiental. Una gran limitación es el carácter errático de la política exterior de Brasil que resulta hasta cierto punto impredecible: el pragmatismo de Itamaraty puede verse estropeado por la diplomacia presidencial de Bolsonaro, tal como ha sucedido en la relación de Brasil con otras potencias como China o Francia. La pérdida de un aliado esencial como Trump para 
Bolsonaro fuerza a Biden a elaborar una nueva estrategia de acercamiento que evite la confrontación y al mismo tiempo ejerza suficiente presión sobre Brasilia.

Es importante mencionar el rol que las otras dos potencias latinoamericanas, México y Argentina, pueden desempeñar en los próximos años. En ambos países gobiernan líderes de izquierda que han entablado una relación muy estrecha y que los ha motivado a llevar a cabo iniciativas conjuntas en busca de lograr una mayor autonomía. Dos ejemplos son llamativos: el liderazgo político al interior del Grupo de Puebla, un foro de concertación de partidos y movimientos de izquierda latinoamericana, que busca articular distintas iniciativas y compartir información y experiencias entre ellos. Por otro lado, los esfuerzos conjuntos por producir y envasar vacunas del laboratorio Astrazeneca para facilitar su acceso en la región es visto por ambos países como un medio para lograr una mayor independencia y autonomía regionales (Télam, 25 de mayo 2021). El diálogo entre los presidentes López Obrador y Fernández refleja un grado de coordinación política entre potencias regionales de izquierda no visto desde la década anterior. Asimismo, resulta un intento importante para ambos países, sobre todo a Argentina, establecer un contrapeso a un Brasil volcado a la derecha y con una política exterior agresiva hacia algunos de sus vecinos como Venezuela y Bolivia.

Así, Estados Unidos enfrenta una región en donde las principales potencias regionales poseen distintas visiones y proyectos sobre la región, y que ejercen medidas de balance suave entre sí, es decir contención a través de medios no militares. Existen, aun así, posibilidades para el manejo de una agenda bilateral con cada uno de ellos. Por ejemplo, México es un aliado fundamental para Estados Unidos en términos económicos, por la vinculación de sus mercados en el marco del T-MEC, y de seguridad y por su rol para contener el avance de la migración centroamericana. La política exterior de Trump fue bastante exitosa en manejar ambos asuntos con López Obrador, lo cual resulta llamativo debido a la estigmatización y el uso de México como chivo expiatorio que llevó a cabo Trump al interior de su país. Una hipótesis convincente es que, además de la afinidad política entre ambos líderes, la política no intervencionista de Trump contribuyó a un manejo bastante pragmático de la relación, considerando la importancia que le otorga López Obrador a la agenda doméstica.

Un punto adicional es el referido a la contención de Venezuela en la región. Debido a la crisis económica del país, Venezuela ha dejado de representar una amenaza regional y por lo tanto una prioridad para la agenda estadounidense en la región. Aun así, persisten problemas de seguridad, corrupción y violaciones de derechos humanos que Estados Unidos ha buscado manejar con el apoyo de sus aliados en la región. La relación con Colombia fue privilegiada durante los gobiernos de Bush, Obama 
y Trump para ejercer un contrapeso en términos políticos y militares a Venezuela. En los últimos años, al igual que con Bolsonaro y López Obrador, Trump y Duque desarrollaron una relación política muy cercana: este tipo de afinidades, sobre las que se ha asentado la política exterior estadounidense, representan desafíos para Biden, quien debe replantear las relaciones sin poner en riesgo lo avanzado por la administración anterior.

\section{China y su presencia creciente en la región}

Finalmente, la creciente influencia de China, otra potencia global con claros intereses expansivos en base a su poder económico es otro de los procesos decisivos para la política regional. Históricamente, Estados Unidos ha ejercido una política de control político y económico sobre la región con el objetivo de evitar la intromisión de actores extrarregionales como la Santa Alianza en el siglo XIX o la Unión Soviética en el siglo XX. El economic statecraft de China en América Latina es un fenómeno reciente que ha concitado mucho interés académico, examinando su efectividad para colocar a la región en la órbita del país asiático (Flores Macías y Kreps 2013; Struver 2016). La evidencia sugiere que América Latina está aun lejos de ir al ritmo de la política exterior China y que no es posible hablar de una transición hegemónica, pero sí ha llenado un vacío importante dejado por Estados Unidos en el plano económico y político, con consecuencias importantes para el ejercicio de su política exterior (Urdinez et al., 2016). En la actualidad China es el principal socio económico de muchos países latinoamericanos, es una de las mayores fuentes de inversión extranjera directa y uno de los prestamistas más importantes en la región (Blanchard, 2018; Jenkins, 2019). Así, el incremento de la presencia china supone un desafío significativo para la proyección de la política exterior de Estados Unidos en tanto su capacidad de ejercer influencia o presión sobre los países de la región disminuye.

Los intereses políticos de China en la región se han manifestado de muchas maneras. China ha buscado fortalecer sus relaciones diplomáticas con los países latinoamericanos para fortalecer la cooperación a distintos niveles, construir un mercado para sus productos y tener acceso a materias primas (Quispe, 2020). Al mismo tiempo, China ha buscado generar una coalición de apoyo en foros internacionales. Por ejemplo, el incremento de las relaciones económicas ha conllevado a un creciente alineamiento de políticas exteriores en ámbitos multilaterales como la Asamblea General de Naciones Unidas (Flores-Macías y Kreps, 2013; Struver, 2016). Asimismo, China ha buscado incluir a América Latina en la Iniciativa de la Franja y la Ruta, un megaproyecto de infraestructura de proyección global, que busca integrar a distintas regiones del mundo. Algunos países de la región se han sumado explícitamente al proyecto mientras que otros permanecen escépticos. Finalmente, China ha buscado promover su 
política de Una Sola China en América Latina con el fin de evitar el reconocimiento de Taiwán (Urdinez y Long, 2021). Todo esto evidencia la importancia estratégica que tiene América Latina para China y por qué es una de sus prioridades en su política de proyección global.

Lo anterior representa un desafío significativo para los Estados Unidos pues se contrapone a su agenda regional: América Latina no es solo la región donde más influencia ha ejercido en el pasado, sino que es la región de mayor proximidad geográfica, lo cual eleva su valor en términos estratégicos. Un ejemplo de ello es la campańa que Estados Unidos ha llevado a cabo para evitar que los países sudamericanos se adhieran al proyecto de la Franja y la Ruta, con visitas directas del entonces secretario de Estado Mike Pompeo a países como Perú en abril de 2019, entre otros. La competencia no ha adquirido una dimensión de confrontación directa en vista de que la presencia de China se ciñe a su propia doctrina de crecimiento pacífico, sin mostrar hasta el momento una postura agresiva o revisionista. Sin embargo, su presencia sí dificulta la agenda Biden para los próximos ańos pues reduce la capacidad de ejercer presión sobre los países latinoamericanos con el fin de alterar sus intereses y comportamiento, así como para influir en ellos y promover un acercamiento. Dos ejemplos actuales ayudan a ilustrar ambas situaciones.

Un ejemplo importante de esto es el manejo de la crisis en Venezuela. Las distintas sanciones económicas de los gobiernos de Obama y Trump han debilitado al régimen de Nicolás Maduro, pero no han sido suficientes para provocar su derrumbe. En los primeros de su gobierno Biden ha flexibilización algunas de las sanciones impuestas por su antecesor, pero no ha dado signos de levantarlas en su totalidad. Blinken ha denominado «dictador brutal» al presidente venezolano y ha apelado a la negociación con los países del Grupo de Lima para continuar con la presión sobre el país caribeño. Sin embargo, las sanciones impuestas previamente han demostrado ser poco eficaces pues no han logrado debilitar al régimen ni quebrar la cohesión interna en torno al dictador. Una explicación plausible radica en el soporte económico que Venezuela obtiene de China. El país asiático no solo es ahora uno de los principales compradores de petróleo, sino que es el principal prestamista del país con una deuda que asciende a más de 60000 millones de dólares a través del Banco de Desarrollo de China (Ferchen, 2018).

El gobierno de Donald Trump identificó la relación entre China y Venezuela como un ejemplo más del debt-trap diplomacy que China aplicaría con países de África y Asia; sin embargo, es importante distinguir la capacidad de agencia de Venezuela en la relación. Mijares (2017) sugiere que existe una estrategia de diversificación de las relaciones exteriores desde el gobierno de Hugo Chávez para lograr una mayor autonomía y, en términos explícitos, contener la presión de parte de Estados Unidos. 
La diversificación externa de compradores, así como de proveedores de armamento (en donde cabe también Rusia) ha permitido al gobierno venezolano contar con recursos para mantener cohesionada a la élite política y militar, limitando así la presión proveniente de Estados Unidos, la Unión Europea y el Grupo de Lima. En este contexto, la política exterior de Biden se ve complicada por la presencia de China en la región como proveedora de recursos.

Un segundo aspecto que ilustra esta dinámica es el estrechamiento de los vínculos de los países latinoamericanos con China a raíz de la pandemia. A las pocas semanas de iniciada la pandemia en América Latina China impulsó, una diplomacia de asistencia ofreciendo mascarillas, ventiladores, pruebas, entre otros. Asimismo, China se ha vuelto el principal suministrador de vacunas en la región a través de empresas como Sinopharm y Sinovac, además de cooperar con países como Brasil en la producción de vacunas. Por otro lado, Urdinez (2021) estudiando el caso de Chile, observa que la cooperación no ha sido centralizada por los estados latinoamericanos, sino que ha dado a través de múltiples canales, en contacto directo con gobiernos provinciales, locales, fundaciones, empresas, entre otros. La pandemia ha representado así una oportunidad excelente para que China despliegue su poder blando en la región, reforzando sus vínculos con los países latinoamericanos y limitando la presencia de Estados Unidos, cuya cooperación con la región durante el mismo período es, en comparación, exiguo.

Existen suficientes indicios que sugieren que la competencia entre ambas potencias no es plena en virtud de las limitaciones que tienen ambos países, vinculadas principalmente a sus necesidades domésticas. Además, China priorizaría la competencia por otras regiones como Asia e inclusive Europa. Sin embargo, a diferencia de China, Estados Unidos tiene menos recursos que ofrecer a los países latinoamericanos y su legitimidad luego de cuatro años de una política exterior errática necesita ser reconstruida. Es un desafío para el gobierno de Biden plantear una política exterior que pueda reconstruir sus vínculos y al mismo tiempo lidiar con la competencia China de forma estratégica y evitando una confrontación que perjudique a la región.

\section{Conclusiones}

Este artículo ha buscado resaltar los principales desafíos a nivel político con los cuáles tendría que lidiar Biden en Latinoamérica en los próximos ańos. La amplia agenda de Biden en la región, que va desde asuntos ambientales, migración, corrupción, derechos humanos, entre otros; se encuentra definida por una serie de procesos políticos a nivel regional y subregional, frente a los cuáles Estados Unidos no ha sabido posicionarse con claridad en administraciones diferentes. El retorno de prácticas autori- 
tarias, la competencia y ambiciones de las potencias latinoamericanas y la presencia de China en la región son desafíos que reducen el margen de maniobra de Estados Unidos en la región y su capacidad de influir en ella. Asimismo, representan un desafío para su autoridad como superpotencia en la región. Este artículo ha buscado presentar dichos desafíos y demostrar cómo pueden afectar las ambiciones de la administración Biden en los próximos cuatro años.

Un aspecto importante a considerar, finalmente, es que estos procesos se enmarcan dentro de un conjunto de transformaciones que, en general, viene sufriendo la política internacional: el debilitamiento del orden liberal internacional y el surgimiento de China como potencia global, el backlash autoritario y el fortalecimiento de black knights o promotores del autoritarismo y la competencia entre países por recursos en un contexto de pandemia y desaceleración económica. Estados Unidos también experimenta una serie de transformaciones, siendo la más preocupante el debilitamiento de las instituciones democráticas, la polarización política y el surgimiento de opciones políticas iliberales. Esto tiene implicaciones muy importantes para la Gran Estrategia de Estados Unidos en el mundo y de forma más concreta para su política exterior en Latinoamérica. Los cuatro años de Biden demostrarán si dichas tendencias pueden ser efectivamente manejadas en su favor.

\section{Referencias bibliográficas}

Biden, J. (2019). Why America Must Lead Again. Rescuing U.S. Foreign Policy After Trump. Foreign Affairs. https://www.foreignaffairs.com/articles/united-states/2020-01-23/ why-america-must-lead-again

Blanchard, J. M. (2018). Brazil's samba with China: economics brought them closer, but failed to ensure their tango. Journal of Chinese Political Science, (24), 583-603.

Chappell, B. (26 de marzo de 2021). «Predatory Elite» also bear the blame for migrant crisis, NSC's Juan Gonzalez Say. NPR. https://www.npr.org/2021/03/26/981284187/ predatory-elite-also-bear-the-blame-for-migrant-crisis-juan-gonzalez-says

Ferchen, M. (2018). China, Venezuela and the Illusion of Debt-Trap Diplomacy. CarnegieTsinghua Center for Global Policy. https://carnegietsinghua.org/2018/08/16/ china-venezuela-and-illusion-of-debt-trap-diplomacy-pub-77089

Flemes, D. y Wehner, L. (2012). Drivers of strategic contestation in South America. GIGA working papers, 207. http://www.jstor.org/stable/resrep07658

Flores-Macías, G. y Kreps, S. E. (2013). The foreign policy consequences of trade: China's commercial relations with Africa and Latin America, 1992-2006. The Journal of Politics, 75(2), 357-371.

Freedom House (2021). Freedom in the World 2021. Democracy under Siege. Freedom House. https://freedomhouse.org/report/freedom-world/2021/democracy-under-siege 
Gardini, G. (2010). Proyectos de integración regional sudamericana: hacia una teoría de convergencia regional. Relaciones Internacionales, (15), 11-31.

Gardini, G. (2016). Brazil: what rise of what power? Bulletin of Latin American Research, 35(1), 5-19.

Gramer, R. (2021). Biden Eyes Career Diplomat as Top Envoy for Latin America. Foreign Policy. https://foreignpolicy.com/2021/02/05/biden-latin-america-state-department-brian-nichols-venezuela-cuba-assistant-secretary-of-state-diplomacy-aftertrump/

Levitsky, S. y Way, L. (2015). The Myth of Democratic Recession. Journal of Democracy, (26), 118-131.

Long, T. (2015). Latin America confronts the United States. Asymmetry and influence. Cambridge University Press.

Long, T. (2018). La relación entre Estados Unidos y América Latina y el Caribe en la era Trump. Revista Mexicana de Politica Exterior, (114) 157-175.

Long, T. (2021). Biden's Latin America policy will be constrained more by weak regional leadership than by Florida's electoral politics. The London School of Economics and Political Science. https://blogs.lse.ac.uk/latamcaribbean/2021/01/19/bidens-latinamerica-policy-will-be-constrained-more-by-weak-regional-leadership-than-by-floridas-electoral-politics/\#comment-11894

Long, T. y Urdinez, F. (2021). Status at the margins: why Paraguay recognizes Taiwan and Shuns China. Foreign Policy Analysis, 17(1). http://doi: 10.1093/fpa/oraa002

Lührmann, A. y Lindberg, S. I. (2019). A third wave of autocratization is here: what is new about it? Democratization, 26(7), 1095-1113.

Malamud, A. (2011). A leader without followers? The growing divergence between the regional and global performance of Brazilian Foreign Policy. Latin American Politics and Society, 53(3), 1-24.

Merke, F., Reynoso, D. y Schenoni, L. (2020). Foreign policy changes in Latin America: Exploring a Middle Range Concept. Latin American Research Review. Forthcoming.

Mijares, V. (2017). Soft-balancing the titans: Venezuelan foreign policy strategy toward the United States, Russia and China. Latin American Policy, 8(2), 201-231.

Nagovitch, P. (2020). Where Kamala Harris Stands on Latin America. AS/COA. https:// www.as-coa.org/articles/where-kamala-harris-stands-latin-america-policy

Quispe, J. (2020). Relaciones entre China y América Latina: variables explicativas para el alineamiento de la política exterior (2003-2018) [tesis de maestría, Centro de Investigación y Docencia Económicas]. http://repositorio-digital.cide.edu/handle/11651/4302

Romano, S. (2018). Estados Unidos y las elecciones en Honduras. En C. Villacorta y E. De Gori (Eds.), Golpe electoral y crisis politica en Honduras (pp. 105-112). CLACSO.

Schenoni, L. 2017. Sub systemic Unipolarities? Power Distribution and State Behavior in South America and Southern Africa. Strategic Analysis, 41(1), 74-86. 
Solís, J. (2021). Can more U.S money really help Central America's Northern Triangle? Americas Quarterly. https:/www.americasquarterly.org/article/ can-more-u-s-money-really-help-central-americas-northern-triangle/

Struver, G. (2016). What friends are made of: Bilateral linkages and Domestic Drivers of Foreign Policy Alignment with China. Foreign Policy Analysis. 12(2), 170-191.

Télam (25 de mayo, 2021). Fernández: «Esto representa la independencia de poder tener nuestras vacunas». Télam. Agencia Nacional de Noticias. https:/www.telam.com.ar/ notas/202105/555376-alberto-fernandez-lopez-obrador-anuncios-astrazeneca.html

Urdinez, F (2021). China's Improvised Diplomacy Mask in Chile. Carnegie Endowment for International Peace. http://www.jstor.org/stable/resrep31122.4

Urdinez, F., Mouron, F., Schenoni, L. y De Oliveira, A. (2016). Chinese Economic Statecraft and U.S. Hegemony in Latin America: An Empirical Analysis, 2003-2014. Latin American Politics and Society, 58(4), 3-30.

Walt, S. (2019). Who will save the American. Foreign Policy. https://foreignpolicy. com/2019/08/05/who-will-invade-brazil-to-save-the-amazon/

Weyland, K. (2016). Realism under Hegemony: Theorizing the Rise of Brazil. Journal of Politics in Latin America, 8(2), 143-173.

Williams, M. (2014). The United States and Latin America, En J.I. Dominguez y A. Covarrubias (Eds.), Routledge Handbook of Latin America in the World (pp. 199-210). Routledge.

Fecha de recepción: 5 de mayo de 2021 Fecha de aprobación: 27 de agosto de 2021 